\title{
Autoevaluación y prospectiva, un insumo para la planeación: experiencia en una división de Bibliotecas de la Universidad Nacional de Colombia
}

\section{Self-assessment and Foresight, as Input for the Planning: Experience in a Division of}

\section{Libraries at National University of Colombia}

Diana Milena Osorno Alzate * Secretaria de Planeación del Municipio de Girardota, Antioquia, Colombia.

Ángela María Benítez Góez ** División de Bibliotecas de la Universidad Nacional de Colombia, Sede Medellín.

Melissa Juliana Velasco García *** Dirección Académica de la Universidad Nacional de Colombia, Sede Medellín.

\section{Resumen}

Este documento tiene como objetivo exponer los referentes teóricos y resultados de una actividad de autoevaluación y prospectiva realizada en la División de Bibliotecas de la Universidad Nacional de Colombia Sede Medellín que contó con la participación de sus funcionarios. La metodología utilizada responde a la aplicación de herramientas de prospectiva (matriz IGO) para identificar y priorizar elementos estratégicos que inducen a la definición de acciones para la construcción de un plan de acción que favorezca el mejoramiento continuo de la División de Bibliotecas para el periodo 2016-2018. Al final, se concluye que los ejercicios participativos de autoevaluación y prospectiva, como insumos para la planeación, aportan significativamente al mejoramiento de una organización.

Palabras clave: Prospectiva, autoevaluación, planeación, división de bibliotecas de la Universidad Nacional de Colombia Sede Medellín. 


\begin{abstract}
This article aims at setting out the theoretical frameworks and results of a selfassessment and foresight activity carried out in the National University of Colombia, Medellin Campus Division of Libraries, which involved the participation of its staff. The methodology used responds to the application of prospective tools (IGO Matrix) in order to identify and to prioritize strategic elements leading to the definition of actions for the construction of an action plan, which favors the continuous improvement of the Division of Libraries for the 2016-2018 period. In the end, it is concluded that the participatory exercises of self-assessment and foresight, as input for planning, meaningfully contribute to the improvement of an organization.
\end{abstract}

Keywords: Prospective, self-assessment, planning, National University of Colombia; Medellin Campus Division of Libraries.

\title{
I. Introducción
}

Los ejercicios de autoevaluación, con miras a la identificación de acciones para lograr un mejoramiento, le permite a las organizaciones y a las unidades administrativas, adscritas a esta, pensarse hacia un futuro deseado y definir el camino para lograrlo. Estas prácticas son reconocidas y validadas en el contexto administrativo e institucional.

Ahora bien, lograr que el punto de partida para la construcción de un plan de acción se alcance, con los aportes de las personas que trabajan y conocen una unidad productiva, administrativa o académica, es quizá mucho más meritorio en la medida en que se construye desde la experiencia y el conocimiento de quienes, por mucho tiempo, han generado y observado las dinámicas propias de cada uno de los procesos. 
Entendiendo la autoevaluación como un proceso de constante análisis, retroalimentación y evaluación, que tiene por finalidad la identificación de fortalezas y debilidades en la búsqueda del mejoramiento continuo, implementado en la División de Bibliotecas de la Universidad Nacional de Colombia Sede Medellín, se realizó un taller de autoevaluación con la participación de los jefes y del personal adscrito a esa dependencia. El ejercicio permitió la realización de actividades que, de modo implícito, incluyen la aplicación de conceptos de prospectiva estratégica, para lograr la definición de acciones sobre los elementos que, de manera concertada, fueron considerados como estratégicos para lograr un futuro deseado.

En este documento se expone la metodología aplicada durante el taller de autoevaluación, así como los resultados obtenidos en cada una de las actividades que hicieron posible la identificación de acciones para la construcción de un futuro plan de desarrollo.

\section{Marco teórico}

\section{a. La Universidad Nacional de Colombia, EI SINAB y la División de Bibliotecas de la Sede Medellín}

La Universidad Nacional de Colombia, como institución de educación superior, tiene su origen hacia la segunda mitad del siglo XIX (22 de septiembre de 1867), en el escenario propio del radicalismo liberal, cuando se quiso poner límites al principio absoluto de la libertad de enseñanza -principio ese que había permitido la aparición de instituciones universitarias de pésima calidad científica y académica- para introducir la suprema inspección y vigilancia estatal de la instrucción pública (Universidad Nacional de Colombia, 2015).

En la actualidad la Universidad Nacional de Colombia hace presencia en ocho regiones del país, con sedes en Bogotá, Medellín, Manizales, Palmira, Amazonía, Orinoquía, Tumaco y Caribe (Isla de San Andrés). La Sede Medellín está constituida por cinco facultades: Arquitectura, Ciencias, Ciencias Agrarias, Ciencias Humanas y Económicas, Minas (Universidad Nacional de Colombia Sede Medellín, 2015 a). 
Para facilitar a la comunidad académica el acceso a la información se dispone del Sistema Nacional de Bibliotecas (SINAB) que "integra los recursos bibliográficos, el talento humano y la infraestructura física y tecnológica, necesarios para ofrecer información académica, artística, científica y los mejores servicios bibliotecarios" (Universidad Nacional de Colombia. SINAB, 2016).

Este sistema de bibliotecas está conformado por divisiones de bibliotecas en cada una de las sedes. A su vez, la División de Bibliotecas de la sede Medellín está constituida por tres bibliotecas, a saber, la biblioteca Efe Gómez, la biblioteca Hernán Garcés González y, además, "posee una colección de Ingeniería Mecánica, ubicada en el Núcleo del Río" (Universidad Nacional de Colombia Sede Medellín, 2015 b).

\section{b. La prospectiva y la autoevaluación}

La prospectiva como concepto se origina en la década de 1950, a partir de los estudios y planteamientos expuestos por los franceses Bertrand de Jouvenel y Gaston Berger. Este último, es considerado el padre de la prospectiva, quien la define como "un haz de luz que, al iluminar el futuro, tiene la propiedad de esclarecer la acción del presente" y complementa diciendo que "la prospectiva es una actitud del espíritu para vislumbrar el futuro" (Berger, 1964, p. 8).

En ese sentido, la prospectiva es definida como una reflexión sobre el futuro a largo plazo, que busca conocer las condiciones de la acción presente sobre las cuales decidir para obtener buenos resultados en el futuro, a largo plazo (Berger, 1957, p. 2). Este pensador sostiene, además, que la prospectiva "en lugar de ser un método o una disciplina, implica una actitud" (Berger, 1959, p. 20). Él afirma que la prospectiva se ocupa del largo plazo y de la 'previsión' del corto plazo (Berger, 1964, p. 8).

En esa dirección, la prospectiva se asocia a una reflexión sobre el futuro, sobre el devenir. Se infiere, entonces, que la prospectiva es una reflexión acerca del porvenir vislumbrado a largo 
plazo, cimentado y fundamentado en las acciones del presente. La prospectiva, planteada por Berger, permite explicar que es necesario conocer los procesos, los métodos y saber cómo operan las diversas dinámicas de las instituciones y las empresas, mirando más allá de las apariencias y de lo externo. Puede decirse, entonces, que la prospectiva entraña también unos criterios analíticos y una visión objetiva sobre lo que acontece en el presente, para poder capitalizar una buena planeación hacia el futuro. En prospectiva es importante que se abran espacios de participación y discusión que permitan esclarecer situaciones, para llegar a consensos que faculten visualizar futuros posibles, deseables y viables. A este respecto se manifiesta que

El futuro no puede ser construido con las imágenes y la visión de una sola persona; tiene que ser construido con la visión de todos aquellos actores que de una u otra manera forman parte del entorno más inmediato y del futuro (Quiroga como se cita en: Botero, Calle, Echavarría, Galindo, y Osuna, 2014, p. 165).

Esto avala la necesidad de hacer construcciones y elaboraciones de manera colectiva. El concepto de prospectiva plantea, también, la declaración de diferentes futuros posibles, probables y deseables y, por consiguiente, abiertos en forma permanente a la controversia (Medina y Ortegón, 2006, 216). Esta polémica se puede dar en términos de argumentación en espacios propicios que permitan lograr acuerdos para generar sinergias y concretar planes comunes para llegar a consensos que aúnen esfuerzos en pro de lograr esos futuros posibles y deseables. Por otra parte, Michel Godet, miembro de la 'Academia Francesa de Tecnología' y catedrático de Prospectiva Estratégica en el Conservatorio Nacional de Artes y Oficios de París, dice "El futuro hay que construirlo, no predecirlo ni preverlo" (Godet, 2010, p. 1461). En este caso, hace referencia a que se debe afrontar el presente con la mira puesta en el futuro. Al respecto, el mismo Godet (2010), expone lo siguiente:

Cualquier persona que ignora su pasado no puede prever sus posibles futuros. Las elecciones del pasado dan forma al futuro. De hecho, la mayoría de los eventos que están ocurriendo ya tienen su raíz en el pasado distante. Los proyectos futuros no 
nacen por casualidad de un poco de imaginación, sino de los deseos surgidos de una herencia cultural y familiar. (p. 1458)

Según estos planteamientos, se puede decir que en prospectiva el futuro no lo construye el hombre aislado, tomado en singularidad, sino el conjunto de los actores sociales o grupos humanos que se unen en el intercambio social para defender sus intereses y obrar utilizando el grado de poder o de solidaridad que cada uno puede ejercer (Peralta y Herrera, 2012, p. 62). En ese sentido, las organizaciones acuden a ejercicios de prospectiva que permiten la "reflexión colectiva" (Godet y Durance, 2011, p. 36), para planear el futuro, teniendo en cuenta sus particularidades, necesidades y expectativas, en la tónica de emprender acciones hacia el futuro, con base en las tendencias y comportamientos de la economía y la sociedad, tanto en lo público como en lo privado.

De esa manera, se dice que la prospectiva "es posible aplicarla a diversas áreas del conocimiento, incluidas las ciencias sociales. $Y$ esa prospectiva se vale y se apoya en diversas disciplinas, técnicas y estrategias para el análisis de un sistema, grupo o comunidad académica" ( (Miklos y Tello, 1997, p. 43) como se cita en: Flórez-Peña, 2013, p. 299). De igual manera, "la prospectiva es también un arte que necesita talentos para ejercerla de una forma no conformista, con intuición y sentido común" (Godet, 2000, p. 33). En contraste, "la prospectiva parte del futuro; concentra la atención sobre el porvenir, imaginándolo a partir del futuro y no del presente" (Miklos y Tello, 2007, p. 16). Es una herramienta que permite planificar, a la vez que posibilita minimizar incertidumbres, sin conferir al azar y las contingencias la batuta de una organización o institución.

En otros términos "la planeación prospectiva, es un proceso que se dirige a la producción de uno o más futuros deseados, que solo ocurrirá si se actúa en el presente" (Del Río y Contreras, 2014, p. 2018). Es por ello, un camino distinto, alternativo al vaticinio o a las predicciones en términos adivinatorios. Los ejercicios de prospectiva generan insumos y propuestas para la planeación que contribuyen a la dinámica y al logro de objetivos en la organización o institución, al tiempo que es una forma de generar procesos participativos 
donde los diversos actores puedan promover y proponer nuevas estrategias para el mejoramiento permanente; entendiendo la estrategia "como un instrumento que favorece el proceso de adaptación de la organización al entorno, de forma evolutiva" (Montoya, 2010, p. 48).

De esta manera, se pueden configurar planes prospectivos encaminados a evitar que las organizaciones caigan en visiones inmediatistas, cortoplacistas y de espontaneísmo, definiendo más bien estrategias y mecanismos para planear el devenir, estableciendo prescripciones proactivas desde el presente, donde se puedan anticipar pronósticos y escenarios con un alto porcentaje de factibilidad. A ese respecto, "el verbo 'anticipar' tiene dos sentidos: a) detectar los cambios que se avecinan y b) diseñar el futuro que anhelamos" (ILPES y Universidad de la Sabana, 2011, p. 2). Se puede decir, por ende, que "gracias a los métodos prospectivos y la planeación de escenarios, es posible construir el futuro más deseable y probable, a partir del presente" (Araujo-Lobo, 2010, p. 1). Pues, "la planeación prospectiva hace parte de las propuestas metodológicas cuya noción central es el estudio del futuro" (Flórez-Peña, 2013, p. 299).

Ahora bien, pasando al ámbito de la autoevaluación, es conveniente señalar que esta es "un proceso amplio y abarcador por medio del cual la institución, en su conjunto, se evalúa a sí misma introspectivamente, con lealtad y objetividad" (Ayarza, 1993, p. 60), con el fin de analizarse internamente para definirse en el presente con miras a lo que desea ser en el futuro. En consecuencia, la autoevaluación se puede definir también como "un proceso de revisión continua, sistemática y organizada, que hace la propia institución de sí misma, o de algunos de sus componentes, de manera integral y participativa" (Ayarza y González, 1994, p. 29). La autoevaluación se basa en hechos y opiniones manifestados por los integrantes de la organización, en espacios propiciados para la reflexión, la crítica constructiva y la argumentación colectiva, con el fin de avanzar y generar insumos para la toma de decisiones y la planeación a largo plazo. Cabe señalar que para el sector educativo se pueden asumir los planteamientos de Arenas y Cabrera (2005, p. 33), que a ese respecto dicen: 
La autoevaluación facilita la adopción de un sistema de control interno o autorregulador, el cual está orientado a garantizar que los procesos de investigación, docencia y extensión logren sus objetivos y que las actividades habituales de todas y cada una de las dependencias se realicen de conformidad con los reglamentos institucionales, sin necesidad de supervisión externa.

Todo lo anterior, permite manifestar que es viable realizar ejercicios de autoevaluación con prospectiva. Para capitalizar esa finalidad, se pueden utilizar diferentes herramientas propias de la prospectiva. Una de ellas es la Matriz IGO (Importancia y Gobernabilidad) que fue implementada por Francisco Mojica, inspirada en la metodología de Michel Godet. Esta herramienta valora las variables "Importancia" y "gobernabilidad". La calificación de "Importancia" es asignada según la pertinencia de la acción con respecto a cada objetivo y la "Gobernabilidad" señala el control que el eslabón de la cadena posee sobre cada variable (Mojica, 2008). Dicha herramienta consiste básicamente en ponderar de 1 a 10 la importancia de las acciones y medir su gobernabilidad entre 0 y 5 , según sea nula (0), débil (1), moderada (3) o fuerte (5) y, posteriormente, llevar estos valores a un plano cartesiano, cuyos ejes son las medianas de los valores de importancia y gobernabilidad, los puntos o coordenadas obtenidas con la "Importancia" como ordenadas y la "Gobernabilidad" como abscisas (Sánchez, Montoya, y Montoya, 2013, p. 51).

\section{Metodología}

Para lograr la identificación de los elementos estratégicos y la definición de acciones concretas en pro del mejoramiento continuo de la División de Bibliotecas, se realizó un ejercicio participativo basado en la metodología o herramienta de trabajo IGO.

En la figura 1 se resumen las actividades abordadas en dicho ejercicio, resaltando que desde este punto se hace referencia al ejercicio de prospectiva como taller de autoevaluación. Es importante destacar que esta metodología implica trabajar en grupos pequeños y lograr un 
solo resultado, en el cual se reflejen los aportes de los grupos y, por ende, de todos los participantes en la actividad.

Figura 1

Modelo de trabajo propuesto
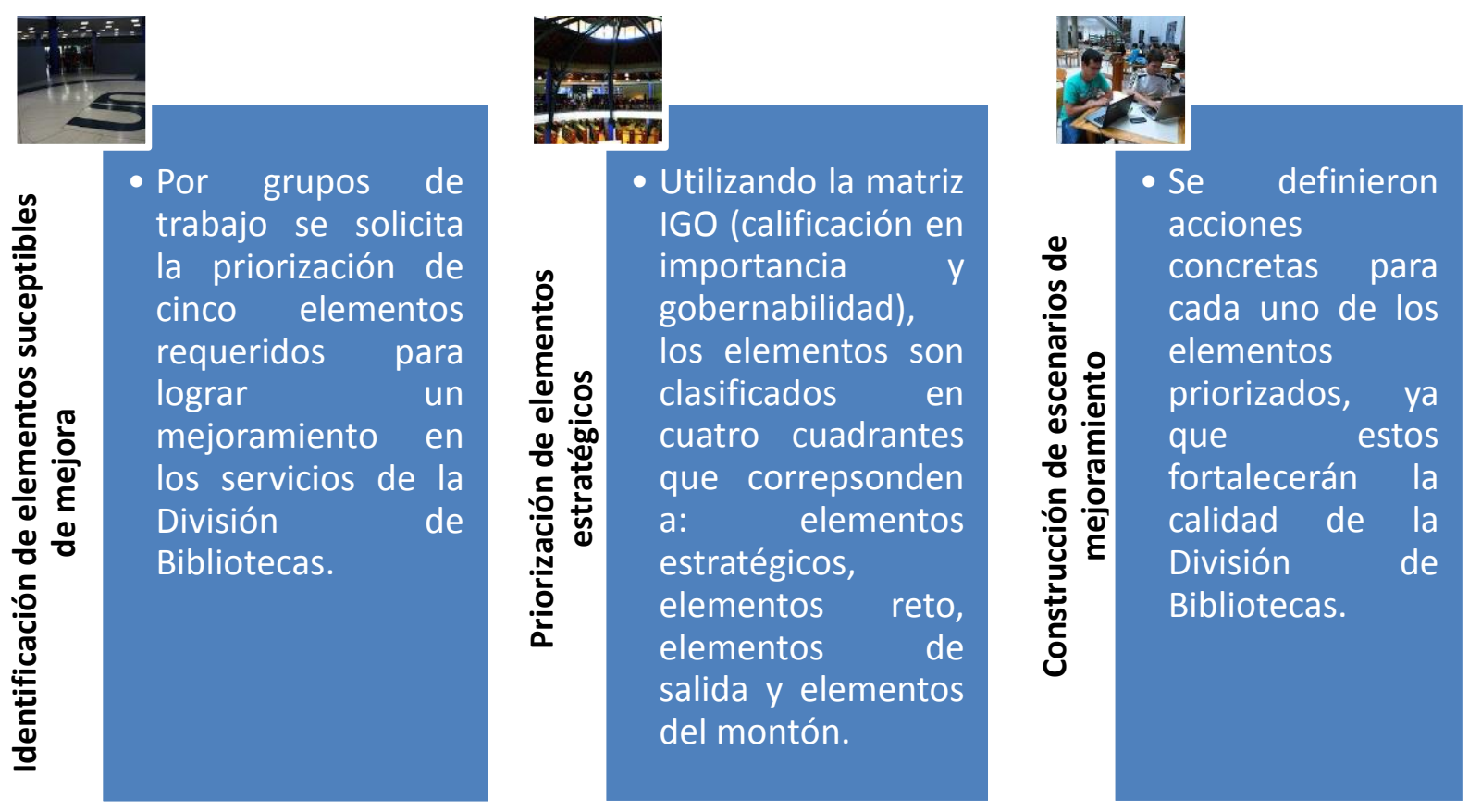

Fuente: Elaboración propia de las autoras.

Con respecto a la metodología, es importante precisar que los resultados se clasifican en cuatro grupos:

- Elementos estratégicos: corresponde a aquellos elementos con un alto valor en importancia y en gobernabilidad. Esta última es entendida como la injerencia que tiene el grupo de trabajo para generar un cambio en la situación descrita.

- Elementos reto: corresponde a aquellos elementos con un alto valor en importancia, pero un bajo valor en gobernabilidad.

- Elementos del montón: en este cuadrante se ubican aquellos elementos que son poco importantes y poco gobernables para el grupo de trabajo. 
- Elementos de salida: como su nombre lo indica, en este cuadrante se ubican elementos que tienen una baja importancia en el mejoramiento de la dependencia, pero que dado su alto valor en gobernabilidad no vale la pena estructurar escenarios de mejora, pues los cambios en dichos escenarios dependerán de instancias diferentes a la División de Bibliotecas.

\section{Resultados - taller de autoevaluación con prospectiva}

A continuación se exponen, por actividad y manteniendo los aportes de los grupos, los resultados del taller de autoevaluación con prospectiva realizado con el personal de la División de Bibliotecas de la Universidad Nacional de Colombia Sede Medellín, en Junio del 2015.

\section{a. Resultados de la actividad 1: identificación de elementos susceptibles de mejora}

Buscando la pluralidad e integración de diversas ópticas, cada uno de los grupos de trabajo establecidos, estuvo conformado por funcionarios de la División de Bibliotecas de la Universidad Nacional de Colombia Sede Medellín. La primera de las actividades correspondió a la identificación de los elementos más importantes por grupos de trabajo, para el mejoramiento de los servicios que ofrece la dependencia. Adicionalmente, se les pidió realizar una descripción de cada elemento.

Es de resaltar que para lograr esta primera depuración de elementos (depuración se refiere a que cada equipo de trabajo debía presentar solo cinco elementos), cada grupo debió realizar plenarias internas utilizando técnicas, como la lluvia de ideas.

En los Cuadros 1, 2, 3 y 4, se presentan los elementos identificados por grupo de trabajo, como los más importantes para el mejoramiento de los servicios que ofrece la División de Bibliotecas, así como la descripción de cada elemento. 


\section{Cuadro 1}

Elementos identificados por el Grupo 1 que favorecerán el mejoramiento de los servicios de la División de Bibliotecas

\begin{tabular}{|c|c|}
\hline Elemento & Descripción \\
\hline $\begin{array}{c}\text { Formación para } \\
\text { empleados }\end{array}$ & $\begin{array}{l}\text { - Actualización y capacitación permanente para los } \\
\text { funcionarios de la biblioteca, en temas relacionados con } \\
\text { adaptación al cambio, planeación, innovación, } \\
\text { comportamiento del consumidor (usuario), atención al } \\
\text { usuario con necesidades especiales, atención a usuarios en } \\
\text { situación de discapacidad, promoción de lectura. }\end{array}$ \\
\hline $\begin{array}{c}\text { Actualización de } \\
\text { plataforma } \\
\text { tecnológica }\end{array}$ & $\begin{array}{l}\text { - Implementación de una plataforma tecnológica que esté a la } \\
\text { vanguardia con el desarrollo de los servicios bibliotecarios } \\
\text { en línea. } \\
\text { - Aumentar las conexiones eléctricas y sistemas de carga de } \\
\text { baterías de computadores portátiles y dispositivos móviles. }\end{array}$ \\
\hline $\begin{array}{l}\text { Optimización de } \\
\text { los espacios }\end{array}$ & $\begin{array}{l}\text { - Adecuación y optimización de los espacios, de acuerdo con } \\
\text { los servicios que se vayan implementando. } \\
\text { - Implementación de un proyecto de centro cultural que } \\
\text { posibilite mayores y mejores espacios para la prestación y } \\
\text { diversificación de servicios orientados al fortalecimiento de } \\
\text { manifestaciones artísticas y culturales. }\end{array}$ \\
\hline $\begin{array}{c}\text { Diversificación de } \\
\text { los servicios }\end{array}$ & $\begin{array}{l}\text { - Aparte de los servicios misionales, se deben ofrecer otros } \\
\text { servicios que contribuyan a la formación integral de los } \\
\text { usuarios y que les posibiliten lugares de esparcimiento y } \\
\text { confort: zonas de descanso, zonas de expresión artística, } \\
\text { zonas de lúdica y juegos. }\end{array}$ \\
\hline Proyección & $\begin{array}{l}\text { - Fortalecimiento de convenios interinstitucionales existentes. } \\
\text { - Generación de nuevos convenios y alianzas con otras } \\
\text { instituciones, en diversos ámbitos. } \\
\text { - Divulgación y comunicación de logros y las fortalezas de la } \\
\text { División de Bibliotecas ante la comunidad en general. } \\
\text { - Expansión de servicios fuera de la biblioteca. }\end{array}$ \\
\hline
\end{tabular}

Fuente: Taller de autoevaluación División de Bibliotecas Universidad Nacional de Colombia - Sede Medellín. Elaboración propia de las autoras. 


\section{Cuadro 2}

Elementos identificados por el Grupo 2 que favorecerán el mejoramiento de los servicios de la División de Bibliotecas

\begin{tabular}{|c|c|}
\hline Elemento & Descripción \\
\hline Normalización & $\begin{array}{l}\text { - Normalizar la información de los diferentes sistemas de } \\
\text { información bibliográfica. } \\
\text { - Facilitar que desde cualquier motor de búsqueda se pueda } \\
\text { ingresar a los recursos bibliográficos. }\end{array}$ \\
\hline Investigación & $\begin{array}{l}\text { - Involucrar la División de Bibliotecas con los procesos de } \\
\text { investigación, ofreciendo servicios especiales (análisis de } \\
\text { indicadores, vigilancia tecnológica y espacios para } \\
\text { investigadores). }\end{array}$ \\
\hline $\begin{array}{l}\text { Ampliación de } \\
\text { horarios }\end{array}$ & $\begin{array}{l}\text { - Ampliar los horarios de los servicios en las Unidades de } \\
\text { información que conforman la División de Bibliotecas. }\end{array}$ \\
\hline $\begin{array}{l}\text { Biblioteca como } \\
\text { tercer lugar }\end{array}$ & $\begin{array}{l}\text { Este elemento se refiere a la biblioteca como lugar prioritario en } \\
\text { la vida de los miembros de la comunidad académica junto con } \\
\text { el hogar y la universidad. En tal sentido la biblioteca debe: } \\
\text { - Ofrecer actividades como talleres artísticos, de } \\
\text { manualidades, cine club y debates desde los diferentes } \\
\text { saberes. } \\
\text { - Lugar de encuentros entre personas dispuestas a enseñar y } \\
\text { otras dispuestas a aprender. } \\
\text { - Lugar de cambio e intercambio cultural. }\end{array}$ \\
\hline $\begin{array}{l}\text { Cualificación del } \\
\text { personal }\end{array}$ & $\begin{array}{l}\text { - Fortalecimiento de capacitaciones a los empleados en } \\
\text { atención al usuario. } \\
\text { - Capacitación técnica y motivacional. }\end{array}$ \\
\hline
\end{tabular}

Fuente: Taller de autoevaluación División de Bibliotecas Universidad Nacional de Colombia - Sede Medellín. Elaboración propia de las autoras. 


\section{Cuadro 3}

Elementos identificados por el Grupo 3 que favorecerán el mejoramiento de los servicios de la División de Bibliotecas

\begin{tabular}{c|c}
\hline Elemento & \multicolumn{1}{c}{ Descripción } \\
\hline $\begin{array}{c}\text { Ampliación de } \\
\text { horario }\end{array}$ & $\begin{array}{l}\text { El servicio mejoraría si se ampliara el horario de 7:00 a.m. - } \\
10: 00 \text { p.m., de lunes a viernes. } \\
\text { Implementación de horario 24 horas en temporadas } \\
\text { específicas durante el semestre académico. }\end{array}$ \\
\hline $\begin{array}{c}\text { Renovación de } \\
\text { equipos y mayor } \\
\text { cobertura }\end{array}$ & $\begin{array}{l}\text { Debido a la obsolescencia de los computadores portátiles } \\
\text { de la Mediateca y a la alta demanda por parte de los } \\
\text { usuarios, se hace necesaria la renovación de estos equipos. }\end{array}$ \\
\hline $\begin{array}{c}\text { Depuración y } \\
\text { digitación de } \\
\text { material } \\
\text { cartográfico }\end{array}$ & $\begin{array}{l}\text { Por dificultades de almacenamiento y de acceso, por parte } \\
\text { de los usuarios al material cartográfico, se sugiere depurar y } \\
\text { digitalizar mapas y planos. }\end{array}$ \\
\hline $\begin{array}{c}\text { Fortalecimiento } \\
\text { en la difusión de } \\
\text { los servicios } \\
\text { bibliotecarios }\end{array}$ & $\begin{array}{l}\text { Mayor difusión de los libros electrónicos y de los servicios a } \\
\text { través de la implementación de estrategias de marketing. }\end{array}$ \\
\hline $\begin{array}{c}\text { Análisis } \\
\text { estadístico }\end{array}$ & $\begin{array}{l}\text { Implementación de análisis estadísticos sobre la demanda y } \\
\text { el uso de los recursos, en aras de mejorar los servicios } \\
\text { ofrecidos a la comunidad académica. }\end{array}$ \\
\hline
\end{tabular}

Fuente: Taller de autoevaluación División de Bibliotecas Universidad Nacional de Colombia - Sede Medellín. Elaboración propia de las autoras. 


\section{Cuadro 4}

Elementos identificados por el Grupo 4 que favorecerán el mejoramiento de los servicios de la División de Bibliotecas

\begin{tabular}{|c|c|}
\hline Elemento & Descripción \\
\hline $\begin{array}{l}\text { Adecuar espacios } \\
\text { físicos dirigidos } \\
\text { al trabajo de } \\
\text { investigadores }\end{array}$ & $\begin{array}{l}\text { - Dirigido a estudiantes de postgrado, docentes e } \\
\text { investigadores. } \\
\text { - Incrementar espacios equipados para el trabajo individual y } \\
\text { de grupos de investigación (espacios con mesas, equipos } \\
\text { de cómputo con acceso a todos los recursos). }\end{array}$ \\
\hline $\begin{array}{l}\text { Ampliar el horario } \\
\text { de servicio }\end{array}$ & $\begin{array}{l}\text { - Lunes a viernes de 6:00 a.m. - 10:00 p.m. y sábados de } \\
\text { 6:00 a.m. - 7:00 p.m. }\end{array}$ \\
\hline $\begin{array}{l}\text { Consulta local de } \\
\text { todos los } \\
\text { recursos }\end{array}$ & $\begin{array}{l}\text { - Que el usuario pueda acceder dentro de la biblioteca a los } \\
\text { recursos en línea sin requerir usuario y contraseña para } \\
\text { "loguearse". }\end{array}$ \\
\hline $\begin{array}{l}\text { Mejoramiento de } \\
\text { infraestructura }\end{array}$ & $\begin{array}{l}\text { - Más disponibilidad de puntos de red y de conexiones } \\
\text { eléctricas. } \\
\text { - Mayor cantidad de equipos de consulta y en mejores } \\
\text { condiciones, sin restricciones de acceso en línea a otros } \\
\text { recursos de información. } \\
\text { - Mejoras en la ventilación de las bibliotecas. }\end{array}$ \\
\hline $\begin{array}{l}\text { Asesoría en la } \\
\text { elaboración de } \\
\quad \text { informes }\end{array}$ & $\begin{array}{l}\text { - Formación y asesoría en normas y pautas para la } \\
\text { elaboración de informes, tesis y artículos científicos. }\end{array}$ \\
\hline
\end{tabular}

Fuente: Taller de autoevaluación División de Bibliotecas Universidad Nacional de Colombia - Sede Medellín. Elaboración propia de las autoras.

\section{b. Resultados de la actividad 2: Priorización de elementos estratégicos}

Previo a la realización de esta actividad, se realizó una consolidación de los elementos susceptibles de mejora, con los aportes de cada grupo. En el cuadro 5 se presentan los elementos comunes y no comunes que el grupo de participantes decidió abordar en las etapas posteriores. 


\section{Cuadro 5}

Elementos identificados, comunes y no comunes, que favorecerán el mejoramiento de los servicios de la División de Bibliotecas

\begin{tabular}{|c|c|}
\hline Elementos & Descripción \\
\hline Formación a empleados & $\begin{array}{l}\text { - Actualización para los funcionarios en temas como } \\
\text { planeación, innovación psicología del usuario, } \\
\text { atención a usuarios y promoción de servicios. }\end{array}$ \\
\hline $\begin{array}{l}\text { Biblioteca como tercer } \\
\text { lugar }\end{array}$ & $\begin{array}{l}\text { - Ofrecer talleres artísticos, debates, cine club, entre } \\
\text { otros. Talleres de estudiantes con estudiantes. } \\
\text { - Retomar el proyecto de centro cultural. }\end{array}$ \\
\hline Ampliación horario & - Lunes a viernes de 7:00 a 10:00 p.m. \\
\hline $\begin{array}{c}\text { Adecuación de espacios } \\
\text { físicos }\end{array}$ & $\begin{array}{l}\text { - Mesas y equipos de cómputo para la investigación } \\
\text { con acceso a todos los recursos. } \\
\text { - Adecuación de los espacios físicos acorde con los } \\
\text { servicios que se van implementado. }\end{array}$ \\
\hline $\begin{array}{l}\text { Actualización de la } \\
\text { plataforma tecnológica }\end{array}$ & $\begin{array}{l}\text { - Actualización acorde con la vanguardia de los } \\
\text { - Incrvicios en línea. } \\
\text { línea. } \\
\text { - Visibilizar los cursos en bases de datos. } \\
\text { - Mejoramiento de equipo de consulta. } \\
\text { - Mejora de la planta física en cuando a conexiones } \\
\text { - Réctricas. }\end{array}$ \\
\hline Normalización & $\begin{array}{l}\text { - Normalizar la información de los diferentes } \\
\text { sistemas de información bibliográfica. Desde } \\
\text { cualquier motor de búsqueda se pueda ingresar a } \\
\text { cualquiera de los recursos bibliográficos. }\end{array}$ \\
\hline
\end{tabular}




\begin{tabular}{|c|c|}
\hline Elementos & Descripción \\
\hline $\begin{array}{l}\text { Depuración y } \\
\text { digitalización de material } \\
\text { cartográfico preguntar si } \\
\text { incluye las tesis }\end{array}$ & $\begin{array}{l}\text { - Hay dificultades en la prestación del servicio físico, } \\
\text { dado el estado del material, ampliación del } \\
\text { mejoramiento en la prestación del servicio. }\end{array}$ \\
\hline $\begin{array}{l}\text { Consulta local de todos } \\
\text { los recursos }\end{array}$ & $\begin{array}{l}\text { - Todos los visitantes deben tener acceso al material } \\
\text { de la Biblioteca institucional. }\end{array}$ \\
\hline $\begin{array}{l}\text { Asesoría en la } \\
\text { elaboración de informes }\end{array}$ & $\begin{array}{l}\text { - Formación y asesoría en normas para la } \\
\text { elaboración de informes de investigación y tesis. }\end{array}$ \\
\hline $\begin{array}{l}\text { Diversificación y difusión } \\
\text { de los servicios }\end{array}$ & $\begin{array}{l}\text { - Llevar los servicios a otros espacios diferentes de } \\
\text { la Biblioteca: visibilizar y expandir los servicios de la } \\
\text { biblioteca. } \\
\text { - Proyección. } \\
\text { - Realizar difusión permanente de libros electrónicos } \\
\text { y servicios, con el fin de generar una cultura de } \\
\text { más uso. } \\
\text { - Fortalecimiento en la difusión de los servicios } \\
\text { bibliotecarios. }\end{array}$ \\
\hline $\begin{array}{l}\text { Análisis estadístico sobre } \\
\text { la demanda y uso de los } \\
\text { servicios }\end{array}$ & $\begin{array}{l}\text { - Realizar un análisis estadístico sobre la demanda y } \\
\text { uso de los servicios para revisar el } \\
\text { aprovechamiento de los recursos de manera tal } \\
\text { que se pueda tomar decisiones. }\end{array}$ \\
\hline
\end{tabular}

Fuente: Taller de autoevaluación División de Bibliotecas Universidad Nacional de Colombia - Sede Medellín. Elaboración propia de las autoras.

La priorización de elementos estratégicos se logró calificando en importancia y en gobernabilidad, cada uno de los elementos anteriores; el resultado de la calificación, permitió clasificar los elementos en alguno de los siguientes cuadrantes:

- Elementos estratégicos - EE.

- Elementos reto - ER.
- Elementos del montón - EM.

- Elementos de salida - ES. 
Los valores obtenidos al considerar la evaluación de los cuatro grupos participantes, se presentan en el cuadro 6:

\section{Cuadro 6}

Calificación en gobernabilidad e importancia, de los elementos comunes y no comunes, que favorecerán el mejoramiento de los servicios de la División de Bibliotecas de la Universidad Nacional de Colombia Sede Medellín

\begin{tabular}{|l|c|c|}
\hline \multicolumn{1}{|c|}{ Elementos } & Gobernabilidad & Importancia \\
\hline EE Formación a empleados & 7,5 & 9,8 \\
\hline ES Biblioteca como tercer lugar & 8,3 & 8,3 \\
\hline ES Ampliación horario & 8,0 & 7,5 \\
\hline EM Adecuación de espacios físicos & 5,3 & 8,0 \\
\hline ER Actualización de la plataforma tecnológica & 6,5 & 9,8 \\
\hline EE Normalización & 8,5 & 9,3 \\
\hline $\begin{array}{l}\text { EM Depuración y digitalización de material } \\
\text { cartográfico preguntar si incluye las tesis }\end{array}$ & 6,8 & 8,3 \\
\hline EM Consulta local de todos los recursos & 7,3 & 7,8 \\
\hline EE Diversificación y difusión de los servicios & 9,3 & 10,0 \\
\hline EM Asesoría en la elaboración de informes & 5,8 & 5,8 \\
\hline EE Análisis estadístico sobre la demanda y uso & 8,5 & 9,8 \\
de los servicios & & 8,55 \\
\hline
\end{tabular}

Fuente: Taller de autoevaluación División de Bibliotecas Universidad Nacional de Colombia - Sede Medellín. Elaboración propia de las autoras. 
Bibliotecas. Vol 35, № 3, julio-diciembre, 2017. EISSN: 1659-3286

URL: $\underline{\text { http://www.revistas.una.ac.cr/index.php/bibliotecas/index }}$

Los elementos de acuerdo con el cuadrante de calificación son los siguientes:

\begin{tabular}{|c|c|}
\hline $\begin{array}{l}\text { Elementos estratégicos: } \\
\text { - Formación a empleados. } \\
\text { - Normalización. } \\
\text { - Diversificación y difusión de los servicios. } \\
\text { - Análisis estadístico sobre la demanda y uso } \\
\text { de los servicios. }\end{array}$ & $\begin{array}{l}\text { Elemento reto: } \\
\text { - Actualización de la plataforma tecnológica. }\end{array}$ \\
\hline $\begin{array}{l}\text { Elementos de salida: } \\
\text { - Biblioteca como tercer lugar. } \\
\text { - Ampliación horario. }\end{array}$ & $\begin{array}{l}\text { Elementos del montón: } \\
\text { - Adecuación de espacios físicos. } \\
\text { - Depuración y digitalización de material } \\
\text { cartográfico preguntar si incluye las tesis. } \\
\text { - Consulta local de todos los recursos. } \\
\text { - Asesoría en la elaboración de informes. }\end{array}$ \\
\hline
\end{tabular}

En la Figura 2 se presenta gráficamente la división por cuadrantes de los elementos.

Figura 2

Gráfico Importancia y Gobernabilidad

IGO

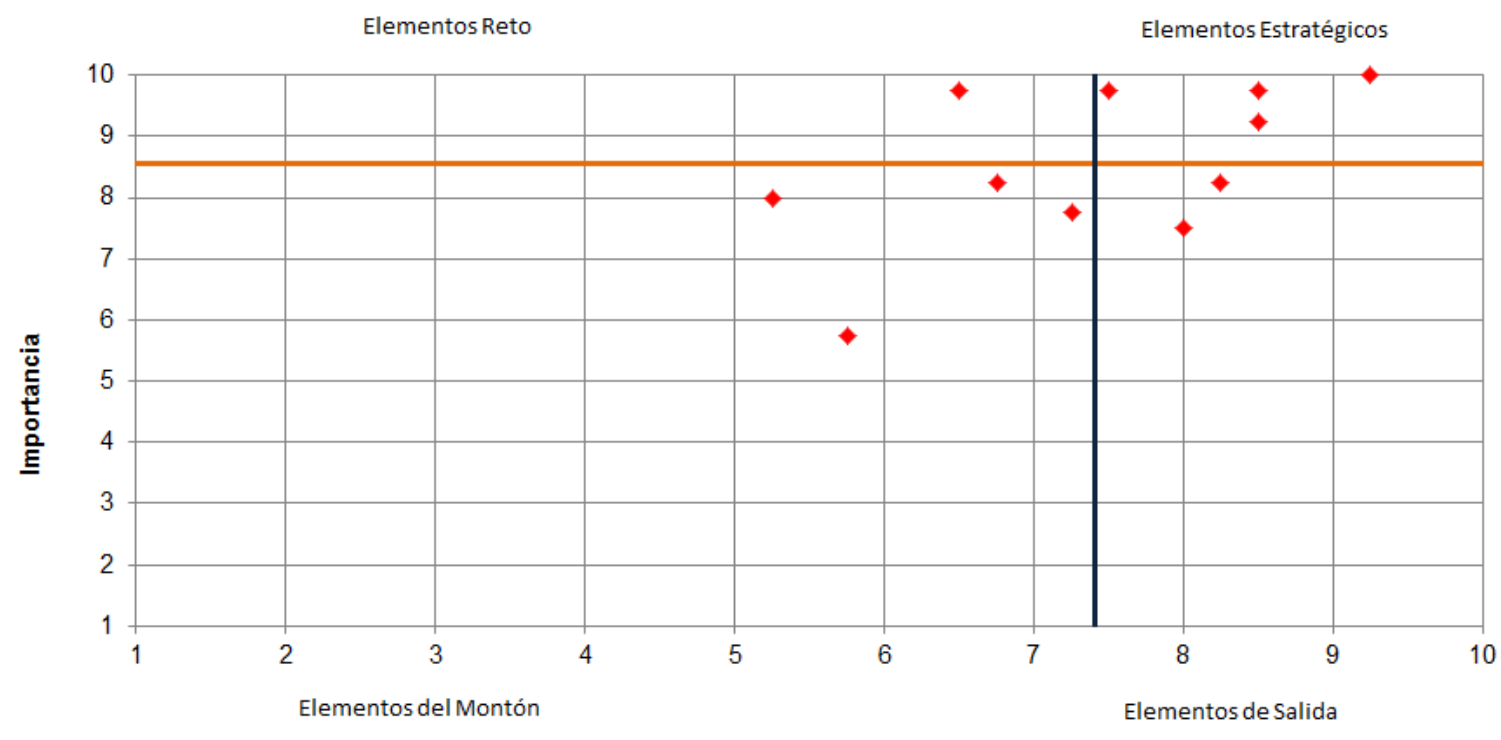

Gobernabilidad

Fuente: Taller de autoevaluación División de Bibliotecas Universidad Nacional de Colombia - Sede Medellín. Elaboración propia de las autoras. 


\section{c. Resultados de la actividad 3: Construcción de escenarios de mejoramiento}

Tal como lo propone Mojica en su artículo "Dos modelos de la escuela voluntarista de prospectiva estratégica" (2008, p. 4) se define "la estrategia como el objetivo y las acciones que son necesarias realizar para lograr el "escenario apuesta". En tal sentido cada grupo identificó acciones para los elementos estratégicos que fortalecerán el mejoramiento de la División de Bibliotecas; dichas acciones se reúnen en un plan de acción que involucra las debilidades y las acciones para transformarlas en fortalezas, además, las metas, los responsables por acción y los recursos necesarios. En los cuadros 7, 8, 9 y 10 se presenta, por elemento y por grupo, las acciones consideradas para alcanzar el escenario apuesta: el mejoramiento de los servicios de la División de Bibliotecas para el trienio 2016-2018.

\section{Cuadro 7}

Acciones propuestas para alcanzar el mejoramiento de los servicios de la División de Bibliotecas, en el elemento: Formación empleados

\begin{tabular}{c|l}
\hline Grupo & \multicolumn{1}{c}{ Acciones propuestas } \\
\hline $\mathbf{1}$ & $\begin{array}{l}\text { Establecer un plan de capacitación: análisis de perfiles de cada empleado (experiencia, } \\
\text { formación, competencias) de acuerdo con la orientación de la dependencia. }\end{array}$ \\
\hline $\mathbf{2}$ & $\begin{array}{l}\text { Capacitación y talleres específicos. } \\
\text { Elaboración y actualización de manuales, guías e instructivos. } \\
\text { Realizar talleres de sensibilización frente a una nueva cultura de Biblioteca. }\end{array}$ \\
\hline $\mathbf{3}$ & $\begin{array}{l}\text { Levantamiento de las necesidades de capacitación de cada área de trabajo. } \\
\text { Gestionar ante la instancia respectiva el apoyo a las capacitaciones programadas. }\end{array}$ \\
$\mathbf{4}$ & $\begin{array}{l}\text { Elaborar el mapa de conocimientos del personal de la Biblioteca. } \\
\text { Transferencia o gestión del conocimiento. } \\
\text { Hacer parte de las capacitaciones dirigidas a empleados administrativos, programadas } \\
\text { por la Oficina de Personal Administrativo. }\end{array}$ \\
\hline
\end{tabular}

Fuente: Taller de autoevaluación División de Bibliotecas Universidad Nacional de Colombia - Sede Medellín. Elaboración propia de las autoras. 


\section{Cuadro 8}

Acciones propuestas para alcanzar el mejoramiento de los servicios de la División de Bibliotecas, en el elemento: Normalización

\begin{tabular}{c|l}
\hline Grupo & \multicolumn{1}{|c}{ Acciones propuestas } \\
\hline $\mathbf{1}$ & $\begin{array}{l}\text { Establecer o definir las políticas. Socializar, difundir y conocer las normas que se van a } \\
\text { aplicar. } \\
\text { Definir un grupo de trabajo. }\end{array}$ \\
\hline $\mathbf{2}$ & $\begin{array}{l}\text { Programar la depuración del catálogo y repositorio a la luz de las normas internacionales. } \\
\text { Capacitar al personal a la luz de las normas. } \\
\text { Realizar un ejercicio de auditoría de la calidad de la información. }\end{array}$ \\
\hline $\mathbf{3}$ & $\begin{array}{l}\text { Normalizar las entradas de autores de la Sede Medellín ajustados a las normas. } \\
\text { Depurar las bases de datos Aleph y el Repositorio Institucional UN de acuerdo con las } \\
\text { normas. } \\
\text { Contratar personal exclusivo para las labores de depuración. } \\
\text { Hacer el registro y revisar los enlaces en Aleph de los recursos en línea. }\end{array}$ \\
\hline
\end{tabular}

Fuente: Taller de autoevaluación División de Bibliotecas Universidad Nacional de Colombia - Sede Medellín. Elaboración propia de las autoras.

\section{Cuadro 9}

Acciones propuestas para alcanzar el mejoramiento de los servicios de la División de Bibliotecas, en el elemento: Diversificación y difusión de los servicios

\begin{tabular}{|c|c|}
\hline Grupo & Acciones propuestas \\
\hline 1 & $\begin{array}{l}\text { Detectar necesidades de servicios a través de grupos focales. } \\
\text { Hacer benchmarking con instituciones pares, nacionales e internacionales. }\end{array}$ \\
\hline 2 & $\begin{array}{l}\text { Diseñar video tutoriales. } \\
\text { Aprovechar los canales de difusión de la información de la Universidad. } \\
\text { Diseñar servicios que fomenten la educación artística y aprovechamiento de espacios de } \\
\text { la Biblioteca. }\end{array}$ \\
\hline 3 & $\begin{array}{l}\text { Trabajar, coordinadamente con Unimedios de la Sede, el diseño y difusión publicitaria. } \\
\text { Articulación de la difusión de todos los servicios a las redes sociales. }\end{array}$ \\
\hline 4 & Diagnóstico e identificación de servicios. \\
\hline
\end{tabular}




\begin{tabular}{l|l}
\hline Grupo & \multicolumn{1}{|c}{ Acciones propuestas } \\
\hline & $\begin{array}{l}\text { Planes de comunicación y difusión / Estrategia de marketing. } \\
\text { Campañas permanentes, en la difusión de servicios en diferentes medios de } \\
\text { comunicación. }\end{array}$
\end{tabular}

Fuente: Taller de autoevaluación División de Bibliotecas Universidad Nacional de Colombia - Sede Medellín. Elaboración propia de las autoras.

\section{Cuadro 10}

Acciones propuestas para alcanzar el mejoramiento de los servicios de la División de Bibliotecas, en el elemento: Análisis estadístico sobre la demanda y uso de los servicios

\begin{tabular}{c|l}
\hline Grupo & \multicolumn{1}{|c}{ Acciones propuestas } \\
\hline $\mathbf{1}$ & $\begin{array}{l}\text { Solicitar asesoría y acompañamiento a la oficina de planeación sobre el análisis e } \\
\text { interpretación de datos. }\end{array}$ \\
\hline $\mathbf{2}$ & $\begin{array}{l}\text { Registro sistemático de actividades y servicios de la Biblioteca. } \\
\text { Análisis periódico de los resultados. } \\
\text { Divulgación de los resultados y toma de decisiones. }\end{array}$ \\
\hline $\mathbf{3}$ & $\begin{array}{l}\text { Implementar un sistema de validación con la huella al ingreso de la Biblioteca. } \\
\text { Analizar la información reportada por los sistemas de información (Aleph) para crear o } \\
\text { mejorar nuevos servicios. }\end{array}$ \\
\hline $\mathbf{4}$ & $\begin{array}{l}\text { Identificación de servicios reales y potenciales. } \\
\text { Encuestas de uso y de impacto. } \\
\text { Propuesta de mejora, proyección y nuevos servicios. }\end{array}$ \\
\hline
\end{tabular}

Fuente: Taller de autoevaluación División de Bibliotecas Universidad Nacional de Colombia - Sede Medellín. Elaboración propia de las autoras.

\section{Conclusiones}

Es valioso realizar un ejercicio de autoevaluación y prospectiva como insumo para la formulación de un plan de acción, ya que la coherencia entre pensar en el futuro y concretar acciones para alcanzarlo, permite optimizar los recursos (especialmente el recurso humano) y alcanzar más fácilmente el mejoramiento que se quiere lograr. 
Resulta significativamente valioso que el ejercicio de autoevaluación con prospectiva que se realizó con el personal de la División de Bibliotecas de la Sede Medellín permita la clasificación de los elementos en estratégicos, reto, de salida y de montón, en el sentido de que se facilita la identificación de los elementos importantes para la institución y en los cuales es posible lograr un cambio.

Planificar de manera concertada y con la participación de quienes, día a día, ejecutan los procesos y tienen contacto permanente con los usuarios, facilita el logro de las acciones definidas para identificar los elementos que requieren mejoramiento.

La participación de los jefes y del personal adscrito a la División de Bibliotecas en un taller de autoevaluación, permite repensar el futuro sobre las lecciones aprendidas para, con base en ello, reflexionar y planear prospectivamente sobre el futuro de la dependencia.

Cuando se hacen ejercicios de autoevaluación con prospectiva resultan elementos que no obtienen una calificación significativa para el total del ejercicio, por lo tanto, no son priorizados ni tomados en cuenta para planeación de futuro. Esto se explica desde la perspectiva del profesor Godet quien manifiesta que "en ejercicios de reflexión, cuando todo el mundo está de acuerdo, debemos sospechar y buscar más de cerca" (Godet, 2010, p. 1459).

Finalmente y como una herramienta de estudio para futuros trabajos, vale la pena dejar planteadas las siguientes preguntas: ¿Son suficientes los aportes de los jefes y el personal adscrito a determinada entidad para la definición de los planes de acción de una organización? ¿Es favorable que en un plan de acción el hecho de que quienes ejecuten las acciones sean los mismos actores que las proponen o se deben tener en cuenta otros actores?

\section{Referencias}

Araujo Lobo, A. (2010). La reflexión prospectiva: elemento clave para la planificación y gestión de la relación universidad-empresa. Omnia, 9 (2), 1-18. Recuperado a partir de http://www.produccioncientifica.luz.edu.ve/index.php/omnia/article/view/7077 
Arenas Hurtado, N., \& Cabrera Dokú, K. (2005). Los procesos de autoevaluación y acreditación y la dinámica de las instituciones. En O. Jaramillo (Ed.), El impacto de los procesos de autoevaluación en la educación superior en Colombia. (p. 154). Bogotá: Convenio Andrés Bello.

Ayarza, H. (1993). Acreditación universitaria en América Latina: antecedentes y experiencias. Santiago, Chile: Centro Interuniversitario de Desarrollo (CINDA): Centro Regional para la Educación Superior en América Latina y el Caribe (CRESALC).

Ayarza, H., y González, L. E. (1994). Política y gestión universitaria. Santiago, Chile: Centro Interuniversitario de Desarrollo - CINDA.

Berger, G. (1957). Sciences humaines et prévision. La Revue des Deux Mondes, 1 (3), $417-$ 426.

Berger, G. (1959). L'attitude prospective. (P. Berger, Gaston, Bourbon-Busset, Jacques de Massé, Ed.). París: Société nouvelle de l'Encyclopédie française. Recuperado a partir de http://www.laprospective.fr/dyn/francais/memoire/texte_fondamentaux/attitudeprospective-g-berger-1959.pdf

Berger, G. (1964). The Prospective Attitude. Management International, 4 (3), 7-10. Recuperado a partir de http://www.jstor.org/stable/40225690

Botero, R. D., Calle, V., Echavarría, M. V., Galindo, R., y Osuna, S. A. (2014). Modelo de laboratorio de prospectiva en gerencia para la Escuela de Ingeniería de Antioquia EIA-. Revista EIA, 10 (9), 161-173. Recuperado a partir de http://www.scielo.org.co/pdf/eia/n19/n19a15.pdf

Del Rio Cortina, J. L., y Julio Contreras, J. del C. (2014). Cruzando el puente : de la selva de la paneación estrategica a la construcción prospectiva de escenarios. Global conference on business and finance proceedings, 9 (2), 2016-2024.

Flórez-Peña, T. del S. (2013). Creación de unidades de formación con apoyo en la planeación prospectiva. Universidad de la Sabana, 16 (2), 297-308. Recuperado a partir de http://educacionyeducadores.unisabana.edu.co/index.php/eye/article/view/2707/3238

Godet, M. (2000). La caja de herramientas de la prospectiva estratégica. (Prospektiker, Ed.) Cuaderno número 5 (4ta. ed.). París: Instituto Europeo de Prospectiva y Estrategia.

Godet, M. (2010). Future memories. Technological Forecasting and Social Change, 77(9), 
1457-1463. http://doi.org/10.1016/j.techfore.2010.06.008

Godet, M., y Durance, P. (2011). La prospectiva estratégica: para las empresas y los territorios. (Unesco, Ed.). París: Dunod, Unesco.

ILPES, y Universidad de la Sabana. (2011). No dejemos que nos siga sorprendiendo el futuro. En CEPAL (Ed.), Prospectiva y estrategia: (compendio de trabajos presentados en el Primer Encuentro de Estudios Prospectivos) (pp. 1-12). Bogotá: CEPAL. Recuperado a partir de http://www.slideshare.net/juanfelipeherrera/prospectiva-y-estrategia

Medina, J., y Ortegón, E. (2006). Manual de prospectiva y decisión estratégica: bases teóricas e instrumentos para América Latina y el Caribe. Santiago de Chile: CEPAL.

Miklos, T., y Tello, M. E. (2007). Planeación prospectiva: Una estrategia para el diseño del futuro. México: Limusa.

Mojica, F. J. (2008). Dos modelos de la Escuela Voluntarista de Prospectiva Estratégica. Universidad Externado de Colombia, 1-11. Recuperado de http://www.franciscomojica.com/articulos/modprosp.pdf

Montoya, I. A. (2010). Una contribución a la comprensión de las estrategias deliberadas y emergentes de las organizaciones, desde una perspectiva evolutiva. Universidad Nacional de Colombia Sede Bogotá.

Peralta, S. M., y Herrera, L. D. (2012). Estudio prospectivo estratégico para el sector solidario en el departamento del Meta hacia el año 2022. Acacías: UNAD.

Sánchez Herrera, B., Montoya Restrepo, I. A., y Montoya Restrepo, L. A. (2013). Aplicación del enfoque integrado de prospectiva y estrategia para el mejoramiento al proceso de selección docente de la Universidad Nacional de Colombia. Innovar, 23, 43-54. Recuperado de http://www.scopus.com/inward/record.url?eid=2-s2.084880840773ypartnerID=40ymd5=38298db5cf5956d89b10d7455071f6c 1

Universidad Nacional de Colombia. Bogotá (2015). Universidad Nacional de Colombia: Historia.

Universidad Nacional de Colombia Sede Medellín. (2015). División de Bibliotecas - Dirección de Bibliotecas Sede Medellín. Recuperado de http://divisiondebibliotecas.medellin.unal.edu.co/

Universidad Nacional de Colombia Sede Medellín, y La Universidad. (2015). Universidad 
Nacional de Colombia: Sede Medellín. Recuperado 21 de mayo de 2015, a partir de http://medellin.unal.edu.co/

Universidad Nacional de Colombia. Bogotá. SINAB. (2016). Acerca del Sistema Nacional de Bibliotecas - SINAB. Recuperado de http://bibliotecas.unal.edu.co/acerca-de/

\section{Notas de las autoras}

* Diana Milena Osorno Alzate: Economista Industrial de la Universidad de Medellín. Especialista en Ingeniería Financiera y Magister en Ingeniería - Ingeniería Administrativa de la Universidad Nacional de Colombia Sede Medellín. Secretaria de Planeación del Municipio de Girardota, Antioquia, Colombia. Correo electrónico: diana.osorno@gmail.com

** Ángela María Benítez Góez: Bibliotecóloga de la Universidad de Antioquia. Especialista en Gestión Empresarial de la Universidad Nacional de Colombia Sede Medellín. Magister en Ingeniería - Ingeniería Administrativa de la Universidad Nacional de Colombia Sede Medellín. Bibliotecóloga de la División de Bibliotecas de la Universidad Nacional de Colombia, Sede Medellín. Correo electrónico: ambenitezg@unal.edu.co

*** Melissa Juliana Velasco García: Ingeniera Industrial y Especialista en Ingeniería Financiera de la Universidad Nacional de Colombia Sede Medellín. Asesora de la Dirección Académica de la Universidad Nacional de Colombia, Sede Medellín. Correo electrónico: mjvelasc@unal.edu.co 